\title{
Totally skew embeddings of manifolds
}

\author{
Mohammad Ghomi · Serge Tabachnikov
}

Received: 27 February 2006 / Accepted: 1 April 2007 / Published online: 12 May 2007

C Springer-Verlag 2007

\begin{abstract}
We study a version of Whitney's embedding problem in projective geometry: What is the smallest dimension of an affine space that can contain an n-dimensional submanifold without any pairs of parallel or intersecting tangent lines at distinct points? This problem is related to the generalized vector field problem, existence of non-singular bilinear maps, and the immersion problem for real projective spaces. We use these connections and other methods to obtain several specific and general bounds for the desired dimension.
\end{abstract}

Keywords Totally skew submanifolds · Tangent developable Skew loop - Generalized vector field problem · Non-singular bilinear maps · Immersion problem for real projective spaces

Mathematics Subject Classification (2000) Primary 53A07 · 57R42; Secondary 57R22

\section{Introduction}

A pair of lines in Euclidean space $\mathbf{R}^{N}$ are said to be skew if they are not parallel and do not intersect. Accordingly, we say that a submanifold $M^{n}$ of $\mathbf{R}^{N}$ is totally skew if every pair of tangent lines of $M$ are skew, unless they are tangent to $M$ at the same point. The simplest

Research of the first author was partially supported by NSF Grant DMS-0336455, and CAREER award DMS-0332333. The second author was supported in part by NSF Grant DMS-0244720 and a BSF grant.

M. Ghomi $(\varangle)$

School of Mathematics, Georgia Institute of Technology, Atlanta, GA 30332, USA

e-mail: ghomi@math.gatech.edu

URL: www.math.gatech.edu/ ghomi

S. Tabachnikov

Department of Mathematics, Pennsylvania State University, University Park, PA 16802, USA

e-mail: tabachni@math.psu.edu

URL: www.math.psu.edu/tabachni 
example is the cubic curve

$$
\mathbf{R} \ni x \longmapsto\left(x, x^{2}, x^{3}\right) \in \mathbf{R}^{3} .
$$

In this paper we construct other examples, while our main focus is the following question: Given a manifold $M^{n}$, what is the smallest dimension $N\left(M^{n}\right)$ such that $M^{n}$ admits a totally skew embedding in $\mathbf{R}^{N}$ ? For instance, the cubic curve, together with the fact that there are no skew pairs of lines in $\mathbf{R}^{2}$, easily yields that $N\left(\mathbf{R}^{1}\right)=3$. In Sect. 2 of this paper we start our investigations by using some classical convexity arguments, together with Thom's transversality theorem, to establish the following basic bounds:

Theorem 1.1 For any manifold $M^{n}$,

$$
2 n+1 \leq N\left(M^{n}\right) \leq 4 n+1 .
$$

Indeed, generically any submanifold $M^{n} \subset \mathbf{R}^{4 n+1}$ is totally skew. Further, if $M^{n}$ is closed, then $N\left(M^{n}\right) \geq 2 n+2$.

In particular, there exist no closed totally skew curves in $\mathbf{R}^{3}$, or, in other words, every immersion of the circle $\mathbf{S}^{1}$ into the Euclidean 3 -space will have a pair of parallel or intersecting tangent lines. On the other hand it is not difficult to show by a direct computation that the curve given by

$$
\mathbf{C} \supset \mathbf{S}^{1} \ni z \longmapsto\left(z, z^{2}\right) \in \mathbf{C}^{2} \simeq \mathbf{R}^{4}
$$

is totally skew [14]. Thus $N\left(\mathbf{S}^{1}\right)=4$. More generally, as we prove in Sect.3, one may explicitly construct totally skew spheres in every dimension:

Theorem 1.2 Let $B: \mathbf{R}^{n+1} \times \mathbf{R}^{n+1} \rightarrow \mathbf{R}^{m}$ be a symmetric non-singular bilinear map. Then

$$
\mathbf{R}^{n+1} \supset \mathbf{S}^{n} \ni x \longmapsto(x, B(x, x)) \in \mathbf{R}^{n+1} \times \mathbf{R}^{m}
$$

is a totally skew embedding. In particular,

$$
N\left(\mathbf{S}^{n}\right) \leq n+m(n)+1,
$$

where $m(n)$ is the smallest dimension where a symmetric nonsingular bilinear map $\mathbf{R}^{n+1} \times$ $\mathbf{R}^{n+1} \mapsto \mathbf{R}^{m}$ exists.

By "symmetric" we mean that $B(x, y)=B(y, x)$, and "non-singular" means that $B(x, y)=0$ only if $x=0$ or $y=0$. In Appendix A we show that the symmetry assumption in the above theorem is not superfluous; in particular, the embedding $\mathbf{S}^{3} \rightarrow \mathbf{R}^{8}$ given by unit quaternions $q \mapsto\left(q, q^{2}\right)$ is not totally skew. Constructing explicit examples of non-singular bilinear maps in Sect.3.3, we obtain

Corollary $1.3 N\left(\mathbf{S}^{n}\right) \leq 3 n+2$, and $N\left(\mathbf{S}^{2 \ell-1}\right) \leq 3(2 \ell-1)+1$.

To obtain more bounds for $N\left(M^{n}\right)$, note that, since $M^{n}$ is locally homeomorphic to $\mathbf{R}^{n}$, any totally skew embedding of $M^{n}$ induces one also of $\mathbf{R}^{n}$. In other words,

$$
N\left(M^{n}\right) \geq N(n):=N\left(\mathbf{R}^{n}\right) .
$$

A number of lower bounds for $N(n)$ are provided by the following result and its corollaries, which are proved in Sects. 4 and 6 respectively. Here $\xi_{n}$ denotes the canonical line bundle over the real projective space $\mathbf{R} \mathbf{P}^{n}$, and $r \xi_{n}$ is the Whitney sum of $r$ copies of $\xi_{n}$. 
Theorem 1.4 If there exists a totally skew disk $D^{n} \subset \mathbf{R}^{N}$, then $(N-n) \xi_{n-1}$ admits $n+1$ linearly independent sections. Thus

$$
N(n) \geq r(n)+n,
$$

where $r(n)$ is the smallest integer such that $r \xi_{n-1}$ admits $n+1$ linearly independent sections.

Finding the number of linearly independent sections of $r \xi_{n}$ is known as the generalized vector field problem. It has been thoroughly studied using various topological methods [1,6$8,10,26,27]$. In particular, [27] provides a table of solutions for $1 \leq p<r \leq 32$. Using these values we immediately obtain:

\section{Corollary 1.5}

\begin{tabular}{|c|c|c|c|c|c|c|c|c|c|c|c|c|c|c|c|c|c|}
\hline$n=$ & 1 & 2 & 3 & 4 & 5 & 6 & 7 & 8 & 9 & 10 & 11 & 12 & 13 & 14 & 15 & 16 & 17 \\
\hline$N(n) \geq$ & 3 & 6 & 7 & 12 & 13 & 14 & 15 & 24 & 25 & 27 & 28 & 31 & 36 & 37 & 38 & 48 & 49 \\
\hline
\end{tabular}

Note that there are significant jumps in the lower bounds in the above table whenever $n$ is a power of 2. Further this table suggests that $N\left(2^{p}\right) \geq 3\left(2^{p}\right)$ for all $p$. The next Corollary of Theorem 1.4, which is based on the vanishing of Stiefel-Whitney classes of $(n+q) \xi_{n-1}$ in dimensions $q$ and higher, shows that this is the case:

Corollary 1.6 Suppose that $N(n)=2 n+q$, with $q \leq n$, and $n+q=2^{r}+m$ with $0 \leq m<2^{r}$. Then $q>m$, and hence $2^{r}-q \leq n \leq 2^{r}-1$. In particular,

$$
N\left(2^{\ell}\right) \geq 3\left(2^{\ell}\right)
$$

since $q=n$ when $n=2^{\ell}$.

The last inequality in the above corollary together with the bound for spheres in Corollary 1.3 yield that

$$
6 \leq N(2) \leq N\left(\mathbf{S}^{2}\right) \leq 8 .
$$

We show in Sect. 5 that, indeed, $N(2)=6$. It is reasonable to expect that, since $\mathbf{S}^{2}$ is a closed manifold, $N(2)<N\left(\mathbf{S}^{2}\right)$, so one might conjecture that $N\left(\mathbf{S}^{2}\right)=7$ or 8 . It would also be interesting to know how often $N(n)$ is equal to $2 n+1$, its lowest possible value. It follows from [6] that this occurs very rarely:

Corollary $1.7 N(n) \geq 2 n+2$, unless $n=1,3$, or 7 .

Recall that $N(1)=3$. Thus excluding the case $n=1$ in the above corollary is not superfluous; however, we do not know whether excluding the other two dimensions are necessary as well. In other words: Does there exist totally skew disks $D^{3} \subset \mathbf{R}^{7}$ or $D^{7} \subset \mathbf{R}^{15}$ ?

The generalized vector field problem is closely related to the immersion problem for real projective spaces, which has been extensively studied too, and a wealth of results is known; see $[9,24]$ for surveys. In particular, one may use the relation between the immersion problem for real projective spaces and nonsingular maps. Then the strong non-immersion theorem of Davis [8] yields:

Corollary $1.8 \mathbf{R P}^{n-1}$ can be immersed in $\mathbf{R}^{N(n)-n-1}$. In particular,

$$
N(2 \ell+1) \geq 2(3 \ell-2 d-\alpha(\ell-d))+3,
$$

where $\alpha(m)$ is the number of 1's in the dyadic expansion of $m$, and $d$ is the smallest nonnegative integer such that $\alpha(\ell-d) \leq d+1$. 
In Appendix B we will also present a sharp result for the existence of totally skew pairs of submanifolds in Euclidean space, which we will prove using two different methods. Next we will mention a number of other related results and motivations for this paper.

Totally skew submanifolds (TS-embeddings) are generalizations of skew loops and skew branes $[13,15,16,33]$ which are submanifolds in an affine space without parallel tangent spaces (S-embeddings). Skew loops were first studied by B. Segre [29] and have connections to quadric surfaces (skew branes were studied by Lai [25]; see also [34]). In particular, the first author and Solomon [15] showed that the absence of skew loops characterizes quadric surfaces of positive curvature (including ellipsoids), while the second author [33] ruled out the existence of skew branes on quadric hypersurfaces in any dimension; see also [36] and [30]. Note, however, that skew loops are only affinely invariant, whereas quadric surfaces are invariant under the more general class of projective transformations. Thus we were led to generalize the concept of skew loops accordingly. This was the original motivation for our paper.

There is also another type of submanifolds, weaker than totally skew, which are defined by requiring that there exist no pairs of intersecting tangent lines at distinct points [14]. The main question of this paper may also be asked for such submanifolds, which we call tangent bundle embeddings (T-embeddings), as well as for skew submanifolds; for example, an $n$-disk cannot be T-embedded in $\mathbf{R}^{2 n}$, see [32]. These questions may be regarded as generalizations of Whitney's embedding problem [37] who proved that every manifold $M^{n}$ may be embedded in $\mathbf{R}^{2 n}$. Of course, TS-embeddings can be viewed as tangent bundle embeddings into real projective spaces.

Finally, we mention a somewhat related notion of $k$-regular embeddings studied in [2-5,18-22,35]. A submanifold $M^{n} \subset \mathbf{R}^{N}$ is called $k$-regular if every $k$-tuple of its distinct points spans a $k$-dimensional vector subspace (there is also an affine version of this notion). For example, it is proved in [5] that there are no $k$-regular embeddings of $\mathbf{R}^{2}$ into $\mathbf{R}^{2 k-\alpha(k)-1}$ where, as before, $\alpha(k)$ is the number of 1's in the dyadic expansion of $k$; when $k$ is a power of 2, this result is the best possible. See [31] for a synthesis and generalization of the notions of totally skew and $k$-regular embeddings, the so called, $(k, l)$-regular maps.

\section{Basic bounds: Proof of Theorem 1.1}

2.1 First we obtain the lower bounds. A pair of affine subspaces $V, W \subset \mathbf{R}^{N}$ are skew provided that every pairs of lines $\ell_{1} \in V$ and $\ell_{2} \in W$ are skew. Let $A G_{n}(N)$ denote the (affine Grassmanian) manifold of the $n$-dimensional affine subspaces of $\mathbf{R}^{N}$, and $G_{n+1}(N+1)$ be the (Grassmanian) manifold of the $(n+1)$-dimensional (vector) subspaces of $\mathbf{R}^{N+1}$. There exists a canonical embedding

$$
i: A G_{n}(N) \rightarrow G_{n+1}(N+1),
$$

given by assigning to each point $p \in \mathbf{R}^{N}$ the line $\ell(p) \subset \mathbf{R}^{n+1}$ which passes through the origin and $(p, 1)$. The following observation is immediate:

Lemma 2.1 Two affine subspaces $V, W \subset \mathbf{R}^{N}$ are skew, if, and only if, the corresponding subspaces $i(V), i(W) \subset \mathbf{R}^{N+1}$ have no nontrivial intersection, that is, $i(V) \cap i(W)=0$. In particular, if $V^{n}, W^{m} \subset \mathbf{R}^{N}$ are skew affine subspaces, then $N \geq m+n+1$.

In particular, no pairs of distinct tangent spaces to a submanifold $M^{n} \subset \mathbf{R}^{2 n}$ are skew. So we conclude that $N\left(M^{n}\right) \geq 2 n+1$. The next observation improves this bound in the case where $M$ is closed, i.e., compact, connected, and without boundary. 
Proposition 2.2 Let $M^{n}$ be a compact manifold, and $f: M \rightarrow \mathbf{R}^{n+k}$ be a continuous map, where $k \geq 2$. Then there exists a pair of distinct points $p, q \in M$ and a hyperplane $H \subset \mathbf{R}^{n+k}$ such that $f(p), f(q) \in H$, while $f(M)$ lies entirely on one side of $H$.

Proof Let $C$ be the convex hull of $f(M)$. If $C$ has no interior points, i.e., it lies in a hyperplane, then we are done. Otherwise $\partial C$ is homeomorphic to $\mathbf{S}^{m}$, where $m=n+k-1>n$. Let $X:=f^{-1}(\partial C)$. If $f \mid X$ is not one-to-one, then we are done, because through each point of $\partial C$ there passes a support plane. Suppose then that $f$ is one-to-one on $X$. Then since $X$ is compact, and $\partial C$ is Hausdorff, it follows that $f(X)$ is homeomorphic to $X$. In particular, $f(X) \neq \partial C$, because $\partial C \simeq \mathbf{S}^{m}$, and $\operatorname{dim}(M)<m$. So there exists a point $r \in \partial C$ such that $r \notin f(M)$. It follows then from Caratheodory's theorem [28] that $r$ must lie in the interior of a simplex $S$ whose vertices are points of $f(M)$. Let $H$ be a support plane of $C$ at $r$. Then $H$ also supports $S$, and therefore $S$ must lie in $H$ because $H$ contains an interior point of $S$. But if $S$ lies in $H$ so do its vertices. So we obtain at least two points of $M$ which lie in $H$.

Suppose that $M^{n} \subset \mathbf{R}^{2 n+1}$ is a closed submanifold. Then, letting $f$ be the inclusion map, Proposition 2.2 implies that there exists a support hyperplane $H$ of $M$ which intersects $M$ at distinct points $p$ and $q$. Since $M$ has no boundary, $H$ must be tangent to $M$ at these points. Thus $M$ has a pair of distinct tangent spaces $T_{p} M$ and $T_{q} N$ which both lie in $H \simeq \mathbf{R}^{2 n}$. So it follows from Lemma 2.1 that $T_{p} M$ and $T_{q} N$ must contain a pair of parallel or intersecting tangent lines. We conclude, therefore, that $N\left(M^{n}\right) \geq 2 n+2$, when $M$ is closed.

2.2 Now we derive the generic upper bound for $N\left(M^{n}\right)$. Since every manifold $M^{n}$ may be easily embedded in $\mathbf{R}^{2 n+1}$ [23], the following result yields that $N\left(M^{n}\right) \leq 4 n+1$.

Proposition 2.3 Any $C^{1}$-immersed submanifold $M^{n} \subset \mathbf{R}^{4 n+1}$ becomes totally skew after an arbitrary small perturbation, and the set of totally skew embeddings $M^{n} \rightarrow \mathbf{R}^{4 n+1}$ is massive in Whitney $C^{\infty}$-topology.

Proof In a nutshell, the argument is very simple. First, embed $M$ into sufficiently large space $\mathbf{R}^{N}$ as a totally skew submanifold. Then one wants to project orthogonally on $\mathbf{R}^{N-1}$ so that the projected manifold remains totally skew. Thus the direction of projection should not be parallel to the affine span of a pair of tangent spaces $T_{p} M$ and $T_{q} M$ for any pair of points $p, q \in M$. The affine span of two tangent spaces is $2 n+1$-dimensional, and a pair $(p, q)$ has $2 n$ "degrees of freedom", hence a desired projection exists as long as $N>4 n+1$.

To make this argument rigorous, one uses Thom transversality theorem, see, e.g., [17,11].

For any $C^{1}$ map $f: M \rightarrow \mathbf{R}^{N}$, and pairs of points $p, q \in M$, the corresponding 1-multijet of $f$ is given by

$$
j_{[2]}^{1} f(p, q):=\left(p, q, f(p), f(q), d f_{p}, d f_{q}\right),
$$

where $d$ is the differential map. The set of all such jets, ranging over mappings $f$ and pairs of points of $M$, is denoted by $J_{[2]}^{1}\left(M, \mathbf{R}^{N}\right)$. Thus we may write

$$
M \times M \ni(p, q) \stackrel{j_{[2]}^{1} f}{\longmapsto} j_{[2]}^{1} f(p, q) \in J_{[2]}^{1}\left(M, \mathbf{R}^{N}\right) .
$$

Let $A \subset J_{[2]}^{1}\left(M, \mathbf{R}^{N}\right)$ be the set of those jets of immersions for which $d f_{p}\left(T_{p} M\right)$ and $d f_{q}\left(T_{q} M\right)$ are not skew. In other words, by Lemma 2.1,

$$
A:=\left\{j_{[2]}^{1} f(p, q) \mid i\left(d f_{p}\left(T_{p} M\right)\right) \cap i\left(d f_{q}\left(T_{q} M\right)\right) \neq 0\right\} .
$$


Then $f(M)$ is totally skew if and only if the jet extension of $f$ is completely disjoint from $A$ :

$$
j_{[2]}^{1} f(M \times M) \cap A=\emptyset .
$$

By Thom's transversality theorem, if $A$ is a stratified subset, then, for a generic $f$, we may assume that $j_{[2]}^{1} f(M \times M)$ is transversal to $A$. Thus once we check that $A$ is stratified, then to establish the above equality, for generic $f$, it would suffice to show that

$$
\operatorname{dim}\left(j_{[2]}^{1} f(M \times M)\right)+\operatorname{dim}(A)<\operatorname{dim}\left(J_{[2]}^{1}\left(M, \mathbf{R}^{N}\right)\right) .
$$

Since $\operatorname{dim}(M)=n$, this is equivalent to

$$
\operatorname{codim}(A):=\operatorname{dim}\left(J_{[2]}^{1}\left(M, \mathbf{R}^{N}\right)\right)-\operatorname{dim}(A)>2 n .
$$

To establish the last inequality, note first that $J^{1}\left(M, \mathbf{R}^{N}\right)$ has the local trivialization $\mathbf{R}^{n} \times L\left(\mathbf{R}^{n}, \mathbf{R}^{N}\right)$ where $L\left(\mathbf{R}^{n}, \mathbf{R}^{N}\right)$ is the space of affine injections $\mathbf{R}^{n} \rightarrow \mathbf{R}^{N}$. One also has a fibering $\pi: L\left(\mathbf{R}^{n}, \mathbf{R}^{N}\right) \rightarrow A G_{n}(N)$, assigning to an affine map its image. Identifying $d f_{p}$ with $i\left(d f_{p}\left(T_{p} M\right)\right)$, we obtain a local projection

$$
\pi \times \pi: J_{[2]}^{1}\left(M, \mathbf{R}^{N}\right) \rightarrow G_{n+1}(N+1) \times G_{n+1}(N+1) .
$$

Let $B \subset G_{n+1}(N+1) \times G_{n+1}(N+1)$ consist of pairs of subspaces in $\mathbf{R}^{N+1}$ with a nontrivial intersection. Then $A=(\pi \times \pi)^{-1}(B)$, and it follows that $\operatorname{codim}(A)$ in $J_{[2]}^{1}\left(M, \mathbf{R}^{N}\right)$ equals $\operatorname{codim}(B)$ in $G_{n+1}(N+1) \times G_{n+1}(N+1)$.

Fix an element of $G_{n+1}(N+1)$, say $\mathbf{R}^{n+1}$. Then almost any $V \in G_{n+1}(N+1)$ is the graph of a linear transformation $\phi: \mathbf{R}^{n+1} \rightarrow \mathbf{R}^{(N+1)-(n+1)}$. Note that $V_{1} \cap V_{2} \neq 0$, if and only if, $\operatorname{rank}\left(\phi_{1}-\phi_{2}\right)<n+1$. Thus $B$ is locally identified with the set of pairs $\left(\phi_{1}, \phi_{2}\right)$ of operators $\mathbf{R}^{n+1} \rightarrow \mathbf{R}^{N-n}$ with $\operatorname{rank}\left(\phi_{1}-\phi_{2}\right)<n+1$. Let $C$ be the set of linear maps $\mathbf{R}^{n+1} \rightarrow \mathbf{R}^{N-n}$ whose rank is less than $n+1$. This is an algebraic variety and is therefore stratified. Further, by the "corank formula" (see, e.g, [17]), the codimension of $C$ is $((N-n)-n)((n+1)-n)=N-2 n$. One has an obvious linear projection $B \rightarrow C$ sending $\left(\phi_{1}, \phi_{2}\right)$ to $\phi_{1}-\phi_{2}$. This projection induces a stratification of $B$, and one concludes that $\operatorname{codim}(B)$ in $G_{n+1}(N+1) \times G_{n+1}(N+1)$ is also $N-2 n$. Thus it follows that $\operatorname{codim}(A)=N-2 n$. In particular, if $N \geq 4 n+1$, then $\operatorname{codim}(A) \geq 2 n+1>2 n$, as desired.

\section{Bilinear maps}

\section{Proof of Theorem 1.2 and its Corollary}

3.1 Let $M \subset \mathbf{R}^{N}$ be a submanifold and $p \in M$. Then by $T_{p} M$ we denote the tangent space of $M$ at $p$, while $\bar{T}_{p} M$ denotes the translation of $T_{p} M$ to the origin, that is

$$
T_{p} M=p+\bar{T}_{p} M .
$$

Lemma 3.1 Let $B: \mathbf{R}^{n+1} \times \mathbf{R}^{n+1} \rightarrow \mathbf{R}^{m}$ be a symmetric bilinear map, $X:=(x, B(x, x))$, and $M:=\left\{X \mid x \in \mathbf{S}^{n}\right\}$. Then,

$$
\bar{T}_{X} M=\left\{(v, 2 B(x, v)) \mid v \in \mathbf{R}^{n+1}, \text { and }\langle v, x\rangle=0\right\}
$$

for every $X \in M$. 
Proof First note that for any $C^{1}$ curve $c:(-\epsilon, \epsilon) \rightarrow \mathbf{R}^{n+1}$,

$$
\begin{aligned}
\left.\frac{d}{d t} B(c(t), c(t))\right|_{t=0} & =\lim _{t \rightarrow 0} \frac{B(c(t), c(t))-B(c(0), c(0))}{t} \\
& =\lim _{t \rightarrow 0} B\left(\frac{c(t)-c(0)}{t}, c(t)\right)+\lim _{t \rightarrow 0} B\left(c(0), \frac{c(t)-c(0)}{t}\right) \\
& =2 B\left(c^{\prime}(0), c(0)\right) .
\end{aligned}
$$

Now suppose that $\|c(t)\|=1$, and define $\gamma:(-\epsilon, \epsilon) \rightarrow M$ by

$$
\gamma(t):=(c(t), B(c(t), c(t))) .
$$

Suppose $c(0)=x$, and set $v:=c^{\prime}(0)$. Then $\gamma(0)=X$,

$$
T_{X} M \ni \gamma^{\prime}(0)=\left(c^{\prime}(0), 2 B\left(c^{\prime}(0), c(0)\right)\right)=(v, 2 B(v, x)),
$$

and it remains to note that, since $\|c(t)\|=1,\langle v, x\rangle=0$.

3.2 Suppose (towards a contradiction) that $M$ is not totally skew. Then, for some distinct $X, Y \in M$, one of the following two conditions holds:

(i) $T_{X} M$ and $T_{Y} M$ contain parallel lines,

(ii) $T_{X} M \cap T_{Y} M \neq \varnothing$.

The above conditions in turn, respectively, imply that

(i) ${ }^{\prime} \quad \bar{T}_{X} M \cap \bar{T}_{Y} M \neq 0$,

(ii) $\quad Y-X \in \bar{T}_{X} M+\bar{T}_{Y} M$.

The implication $(i) \Longrightarrow(i)^{\prime}$ is clear. To see $(i i) \Longrightarrow(i i)^{\prime}$, recall that $T_{X} M=X+\bar{T}_{X} M$, and $T_{Y} M=Y+\bar{T}_{Y} M$. Thus (ii) implies that $X+v=Y+w$ for some $v \in \bar{T}_{X} M$ and $w \in \bar{T}_{Y} M$. Consequently, $Y-X=v-w \in \bar{T}_{X} M+\bar{T}_{Y} M$.

First assume that $(i)^{\prime}$ holds. Recall that, by definition of $M$,

$$
X=(x, B(x, x)), \quad Y=(y, B(y, y)),
$$

for some distinct $x, y \in \mathbf{S}^{n}$. Thus, by Lemma 3.1, there exist non-zero vectors $u, v \in \mathbf{R}^{n}$ such that

$$
(v, 2 B(x, v))=(u, 2 B(y, u)) .
$$

So $u=v$, and $B(x, v)=B(y, u)$. These imply that $B(y-x, v)=0$. Since $B$ is non-singular and $x \neq y$, we conclude that $v=0$, which is a contradiction.

Next assume that (ii $)^{\prime}$ holds. Then, by (2) and Lemma 3.1, there exist $u, v \in \mathbf{R}^{n+1}$ such that $\langle v, x\rangle=0,\langle u, y\rangle=0$, and

$$
y-x=u+v, \quad B(y, y)-B(x, x)=2 B(x, v)+2 B(y, u) .
$$

Substituting $y=x+u+v$ into the second equation in (3) and collecting terms we obtain: $B(u, u)=B(v, v)$. It follows that $B(u+v, u-v)=0$. Thus, since $B$ is non-singular, either $u=-v$ or $u=v$. If $u+v=0$, then, by the first equation in (3), $y=x$, which contradicts the assumption that $x$ and $y$ are distinct. If $u=v$ then $v$ is orthogonal to $x$ and $y$, and, by the first equation in (3), $y-x=2 v$. Taking the inner-product of both sides of this equation with $v$ yields $0=2|v|^{2}$. Thus $v=0$, which is again a contradiction. So we conclude that $M$ is totally skew. 
3.3 Proof of Corollary 1.3

Note that

$$
\left(\left(x_{0}, \ldots, x_{n}\right),\left(y_{0}, \ldots, y_{n}\right)\right) \mapsto\left(\sum_{i+j=0} x_{i} y_{j}, \ldots, \sum_{i+j=2 n} x_{i} y_{j}\right)
$$

gives a nonsingular bilinear map $\mathbf{R}^{n+1} \times \mathbf{R}^{n+1} \mapsto \mathbf{R}^{2 n+1}$. Thus, by Theorem 1.2, we have

$$
N\left(\mathbf{S}^{n}\right) \leq n+(2 n+1)+1=3 n+2,
$$

as had been claimed. Further, (4) may also be viewed as a complex mapping $\mathbf{C}^{n+1} \times \mathbf{C}^{n+1} \mapsto$ $\mathbf{C}^{2 n+1}$, which in turn yields a real non-singular bilinear map $\mathbf{R}^{2 \ell} \times \mathbf{R}^{2 \ell} \mapsto \mathbf{R}^{4 \ell-2}$. Consequently,

$$
N\left(\mathbf{S}^{2 \ell-1}\right) \leq(2 \ell-1)+(4 \ell-2)+1=3(2 \ell-1)+1
$$

Note 3.2 The assumption in Theorem 1.2 that $B$ be symmetric is essential. For instance, the (non-symmetric) non-singular bilinear map $\mathbf{R}^{4} \times \mathbf{R}^{4} \mapsto \mathbf{R}^{4}$ given by quaternion multiplication fails to produce a totally skew embedding of $\mathbf{S}^{3}$ in $\mathbf{R}^{8}$, in contrast to the totally skew embedding of $\mathbf{S}^{1}$ in $\mathbf{R}^{4}$ given by complex multiplication.

Note 3.3 We do not know whether there exist symmetric bilinear non-singular maps $\mathbf{R}^{n+1} \times$ $\mathbf{R}^{n+1} \mapsto \mathbf{R}^{n+q}$ with $q<n$. Construction of such maps would improve the upper bounds for $N\left(\mathbf{S}^{n}\right)$ obtained in this section. The existence of a symmetric bilinear non-singular map $\mathbf{R}^{n} \times \mathbf{R}^{n} \mapsto \mathbf{R}^{n+q}$ also implies that $\mathbf{R P}^{n-1}$ can be embedded in $\mathbf{R}^{n+q-1}$, see [24]. Thus totally skew submanifolds are related to embeddings of real projective spaces (in addition to the immersion problem which will be discussed in Sect. 5.3).

\section{The vector field problem}

\section{Proof of Theorem 1.4}

Let $N:=N(n)$. Then there exists a totally skew disc $D^{n} \subset \mathbf{R}^{N}$. Define the (Gauss) map

$$
D \ni x \stackrel{f}{\longmapsto} i\left(T_{x} D\right) \in G_{n+1}(N+1),
$$

where $i$ is as in (1). By Lemma 2.1, since $D$ is totally skew,

$$
f(x) \cap f(y)=0, \text { for all } x \neq y \text { in } D .
$$

Replacing $D$ by a smaller subset, we may assume that $D$ is the graph of a mapping $\phi: U^{n} \rightarrow$ $\mathbf{R}^{N-n}$, where $U \subset \mathbf{R}^{n}$ is an open disc centered at the origin. Then, for every $x \in D, f(x)$ is the graph of a uniquely determined linear map $A(x): \mathbf{R}^{n+1} \rightarrow \mathbf{R}^{N-n}$.

Note that $f(x) \cap f(y) \neq 0$, if, and only if, $A(x)-A(y)$ has non-zero kernel. Thus $A(x)-A(y): \mathbf{R}^{n+1} \rightarrow \mathbf{R}^{N-n}$ is a linear injection for all $x \neq y$ in $D$. Such mappings may be identified with the (Stiefel) manifold $V_{n+1}(N-n)$ of $(N-n) \times(n+1)$ matrices with linearly independent columns. Hence we obtain a map

$$
\left(D \times D-\Delta_{D}\right) \ni(x, y) \stackrel{F}{\longmapsto} A(x)-A(y) \in V_{n+1}(N-n),
$$


where $\Delta_{D}$ denotes the diagonal elements of $D \times D$. Note that $F$ is antisymmetric, that is,

$$
F(y, x)=-F(x, y) .
$$

After a dilation, we may assume that $\mathbf{S}^{n-1} \subset U$. Thus we may define a mapping

$$
\mathbf{S}^{n-1} \ni u \stackrel{\Phi}{\longmapsto}((u, \phi(u)),(-u, \phi(-u))) \in D \times D-\Delta_{D} .
$$

Now if we set $G:=F \circ \Phi$, we obtain a mapping

$$
\mathbf{S}^{n-1} \stackrel{G}{\longmapsto} V_{n+1}(N-n) .
$$

Note that $\Phi(-u)$ is the transpose of $\Phi(u)$, that is, changing $u$ to $-u$ switches the two components of $\Phi(u)$. This, together with (5), yields that $G$ is odd:

$$
G(-u)=-G(u) .
$$

Next, let $\left\{e_{1}, \ldots, e_{n+1}\right\}$ be the standard basis for $\mathbf{R}^{n+1}$ and, for $1 \leq i \leq n+1$, define the mappings $\sigma_{i}$ by

$$
\mathbf{R P}^{n-1}:=\mathbf{S}^{n-1} / \mathbf{Z}_{2} \ni[u] \stackrel{\sigma_{i}}{\longmapsto}\left[\left(u, G(u) \cdot e_{i}\right)\right] \in\left(\mathbf{S}^{n-1} \times \mathbf{R}^{N-n}\right) / \mathbf{Z}_{2},
$$

where $[*]:=\{*,-*\}$. Note that, using (6), we have

$$
\sigma_{i}([-u])=\left[\left(-u, G(-u) \cdot e_{i}\right)\right]=\left[-\left(u, G(u) \cdot e_{i}\right)\right]=\left[\left(u, G(u) \cdot e_{i}\right)\right]=\sigma_{i}([u]) .
$$

Thus $\sigma_{i}$ is well-defined. Finally, it remains to note that

$$
\left(\mathbf{S}^{n-1} \times \mathbf{R}^{N-n}\right) / \mathbf{Z}_{2} \simeq(N-n) \xi_{n-1} .
$$

So $\sigma_{i}$ is a section of $(N-n) \xi_{n-1}$ over $\mathbf{R} \mathbf{P}^{n-1}$. Further, since $\left\{G(u) \cdot e_{i}\right\}$ is the set of columns of $G(u)$, it is linearly independent by definition, and it follows that $\left\{\sigma_{i}\right\}$ is linearly independent. So we conclude that $(N-n) \xi_{n-1}$ admits $n+1$ linearly independents sections.

\section{A totally skew disk in $\mathbf{R}^{6}$}

As we mentioned in Sect. 1 and Theorem 1.4, which we just proved, immediately yields Corollary 1.5, and, in particular, the bound $N(2) \geq 6$. In other words, the smallest possible dimensions of an affine space which contains a totally skew disk cannot be less than 6 . On the other hand:

Proposition 5.1 The complex cubic curve given by

$$
\mathbf{R}^{2} \simeq \mathbf{C} \ni z \longmapsto\left(z, z^{2}, z^{3}\right) \in \mathbf{C}^{3} \simeq \mathbf{R}^{6}
$$

is a totally skew embedding. In particular, $N(2) \leq 6$.

Proof First we show that the complex cubic curve is totally skew in the complex sense, that is, it has neither intersecting nor parallel complex tangent lines. Indeed, the tangent vector at point $P(z):=\left(z, z^{2}, z^{3}\right)$ is $\left(1,2 z, 3 z^{2}\right)$. If two such vectors are parallel at points $P(z)$ and $P\left(z_{1}\right)$ then $z=z_{1}$. If the tangent lines at $P(z)$ and $P\left(z_{1}\right)$ intersect then

$$
\left(z-z_{1}, z^{2}-z_{1}^{2}, z^{3}-z_{1}^{3}\right)=u\left(1,2 z, 3 z^{2}\right)+v\left(1,2 z_{1}, 3 z_{1}^{2}\right)
$$


for some $u, v \in \mathbf{C}$ (cf. the proof of Theorem 1.2 above). Equating the first components, we have $z-z_{1}=u+v$; equating the second yields $z^{2}-z_{1}^{2}=2 u z+2 v z_{1}$. Hence $(u+v)\left(z+z_{1}\right)=$ $2 u z+2 v z_{1}$, and therefore $(u-v)\left(z-z_{1}\right)=0$. It follows that either $z=z_{1}$ or $u=v$. In the later case equate the third components to obtain $z^{3}-z_{1}^{3}=3 u\left(z^{2}+z_{1}^{2}\right)$, and since $z-z_{1}=2 u$, one has $2 u\left(z^{2}+z z_{1}+z_{1}^{2}\right)=3 u\left(z^{2}+z_{1}^{2}\right)$. It follows that $u\left(z-z_{1}\right)^{2}=0$. Therefore either $z=z_{1}$ or $u=0$, which again implies that $z=z_{1}$.

Suppose now that the 2D-disk is not totally skew. Then either the real tangent lines at some distinct points $P(z)$ and $P\left(z_{1}\right)$ are parallel or they intersect. In the former case, let $\xi$ and $\xi_{1}$ be parallel tangent vectors. Then the vectors $J \xi$ and $J \xi_{1}$ are also parallel where $J$ is the complex structure in $\mathbf{C}^{3}$, the operator of multiplication by $\sqrt{-1}$. It follows that the complex tangent lines at $P(z)$ and $P\left(z_{1}\right)$ are parallel. On the other hand, if the real tangent lines at $P(z)$ and $P\left(z_{1}\right)$ intersect, then so do the complex tangent lines as well. In both cases we obtain a contradiction.

So the above proposition together with Corollary 1.5 yield:

Corollary $5.2 N(2)=6$.

\section{Proof of the other Corollaries of Theorem 1.4}

\subsection{Proof of Corollary 1.6}

The total Stiefel-Whitney class of $(n+q) \xi_{n-1}$ is $(1+x)^{n+q}$, where $x$ is the generator of the homology group $H^{1}\left(\mathbf{R} \mathbf{P}^{n-1} ; \mathbf{Z}_{2}\right)$. Since the Stiefel-Whitney classes vanish in dimensions $q$ and higher,

$$
\left(\begin{array}{c}
n+q \\
i
\end{array}\right)=0(\bmod 2) \text { for } i=q, \ldots, n-1 .
$$

By assumption, $2^{r+1}>n+q=2^{r}+m$ and $n \geq q$. These inequalities imply that $2^{r+1}>2 q$, and hence $2^{r}>q$. It follows that $n-m=2^{r}-q>0$. Thus $m \leq n-1$.

Suppose $q \leq m$. Then $m \in\{q, \ldots, n-1\}$. It is well known that the maximal power of 2 dividing $k$ ! equals $k-\alpha(k)$. It follows that

$$
\left(\begin{array}{c}
2^{r}+m \\
m
\end{array}\right)=1(\bmod 2)
$$

which contradicts (7). So we conclude that that $q>m$.

\subsection{Proof of Corollary 1.7}

In [6], Davis estimated the maximal number of linearly independent sections of the bundles $k \xi_{p}$. In particular, if $\left(\begin{array}{c}k-1 \\ p\end{array}\right)$ is odd then $k \xi_{p}$ has at most

$$
s:=k-p+2 v(k)+\epsilon(v(k), p)
$$

independent sections; here $v(k)$ is the greatest power of 2 dividing $k$, and $\epsilon$ depends on the mod 4 values of its arguments with

$$
\epsilon(0,2)=\epsilon(3,2)=0, \quad \epsilon(1,2)=\epsilon(2,2)=-2 .
$$


Assume that $N=2 n+1$, that is, $q=1$. By Corollary 1.6, $n=2^{r}-1$. So, by Theorem 1.4, $2^{r} \xi_{2^{r}-2}$ is a trivial bundle. Apply the theorem of Davis with $k=2^{r}$ and $p=2^{r}-2$. Then $v(k)=r, p=2 \bmod 4$ and $s=2+2 r$ for $r=0,3 \bmod 4$ and $s=2 r$ for $r=1,2 \bmod 4$. Since $2^{r} \xi_{2^{r}-2}$ has $2^{r}$ sections, one has: $s \geq 2^{r}$, and therefore $2+2 r \geq 2^{r}$, which is clearly impossible unless $r \leq 3$.

\subsection{Proof of Corollary 1.8}

By Theorem 1.4, $(N(n)-n) \xi_{n-1}$ admits $n+1$ linearly independent sections. As we showed in Sect. 4.1, this implies that there exists a $\mathbf{Z}_{2}$-equivariant map $G: \mathbf{S}^{n-1} \rightarrow V_{n+1}(N-n)$. Since $V_{n+1}(N-n)$ may be identified with the space of linear injections $\mathbf{R}^{n+1} \rightarrow \mathbf{R}^{N-n}, G$ induces a mapping $\mathbf{S}^{n-1} \times \mathbf{R}^{n+1} \mapsto \mathbf{R}^{N-n}$ and, by homogeneous extension, a non-singular map

$$
g: \mathbf{R}^{n} \times \mathbf{R}^{n+1} \rightarrow \mathbf{R}^{N-n} .
$$

That is, $g(x, y)=0$ only if $x=0$ or $y=0$. Further, $g$ is homogeneous of degree 1 in each variable: $g(s x, t y)=s t g(x, y)$ for all $s, t \in \mathbf{R}$. Equivalently, one obtains an axial map

$$
\bar{g}: \mathbf{R} \mathbf{P}^{n-1} \times \mathbf{R} \mathbf{P}^{n} \rightarrow \mathbf{R} \mathbf{P}^{N-n-1},
$$

where "axial" means that the restriction of $\bar{g}$ on each factor is homotopic to the inclusion. By restriction, one also has an axial map

$$
h: \mathbf{R} \mathbf{P}^{n-1} \times \mathbf{R} \mathbf{P}^{n-1} \rightarrow \mathbf{R} \mathbf{P}^{N-n-1} .
$$

The existence of such a map is equivalent to the existence of an immersion $\mathbf{R P}^{n-1} \rightarrow$ $\mathbf{R}^{N-n-1}$, see [9,24]. The stated lower bound for $N(n)$ now follows from the immersion theorem of Davis [8].

Note 6.1 The relation between totally skew submanifolds and immersions of projective spaces is rather unexpected. Another, seemingly unrelated problem which turned out to be equivalent to the immersion problem for $\mathbf{R P}^{n}$ concerns the topological complexity of the motion planning problem on $\mathbf{R P}^{n}$ [12].

Acknowledgments It is a pleasure to acknowledge useful discussions with M. Farber, D. Fuchs, M. Kossowski, K. Y. Lam, S. Lvovsky, B. Solomon, and S. Yuzvinsky. Part of this work was completed while the first author was at the University of South Carolina. The second author acknowledges the hospitality of ETH in Zurich where part of this work was done.

\section{Appendix A: Quaternions}

Here we show that in contrast to the embedding $\mathbf{S}^{1} \rightarrow \mathbf{R}^{4}$, given by the unit complex numbers $z \mapsto\left(z, z^{2}\right)$, the quaternionic embedding

$$
H \supset \mathbf{S}^{3} \ni q \longmapsto\left(q, q^{2}\right) \in H^{2} \simeq \mathbf{R}^{8}
$$

fails to be totally skew. In particular, the symmetry assumption in Theorem 1.2 is not superfluous. To see this first note that if $q=(a, b, c, d)$, then the above mapping is given by

$$
\left(a, b, c, d, a^{2}-b^{2}-c^{2}-d^{2}, 2 a b, 2 a c, 2 a d\right) .
$$


The image of this map, say $M$, coincides with $f^{-1}(o)$, where $f: \mathbf{R}^{8} \rightarrow \mathbf{R}^{5}, f=\left(f_{1}, \ldots, f_{5}\right)$, is the submersion defined by

$$
\begin{aligned}
& f_{1}\left(x_{1}, \ldots, x_{8}\right):=1-x_{1}^{2}-x_{2}^{2}-x_{3}^{2}-x_{4}^{2}, \\
& f_{2}\left(x_{1}, \ldots, x_{8}\right):=x_{5}-x_{1}^{2}+x_{2}^{2}+x_{3}^{2}+x_{4}^{2}, \\
& f_{3}\left(x_{1}, \ldots, x_{8}\right):=x_{6}-2 x_{1} x_{2}, \\
& f_{4}\left(x_{1}, \ldots, x_{8}\right):=x_{7}-2 x_{1} x_{3}, \\
& f_{5}\left(x_{1}, \ldots, x_{8}\right):=x_{8}-2 x_{1} x_{4} .
\end{aligned}
$$

The tangent plane of $M$ at $u \in M$ is determined by the system of equations

$$
\left\langle\operatorname{grad} f_{i}(u), x-u\right\rangle=0
$$

$i=1, \ldots, 5$. Explicitly, these are

$$
\begin{aligned}
u_{1} x_{1}+u_{2} x_{2}+u_{3} x_{3}+u_{4} x_{4} & =u_{1}^{2}+u_{2}^{2}+u_{3}^{2}+u_{4}^{2}, \\
-2 u_{1} x_{1}+2 u_{2} x_{2}+2 u_{3} x_{3}+2 u_{4} x_{4}+x_{5} & =2\left(-u_{1}^{2}+u_{2}^{2}+u_{3}^{2}+u_{4}^{2}\right)+u_{5}, \\
2 u_{2} x_{1}+2 u_{1} x_{2}-x_{6} & =4 u_{1} u_{2}-u_{6}, \\
2 u_{3} x_{1}+2 u_{1} x_{3}-x_{7} & =4 u_{1} u_{3}-u_{7}, \\
2 u_{4} x_{1}+2 u_{1} x_{4}-x_{8} & =4 u_{1} u_{4}-u_{8} .
\end{aligned}
$$

A pair of tangent planes of $M$ intersect provided that there exist $u, \bar{u} \in M$, which satisfy the above equations for the same $x \in \mathbf{R}^{8}$. This is the case if

$$
u=(0,1,0,0,-1,0,0,0), \quad \bar{u}=(0,0,1,0,-1,0,0,0),
$$

and

$$
x=\left(0,1,1, x_{4},-1,0,0,0\right) .
$$

So $M$ is not totally skew.

\section{Appendix B: Totally skew pairs}

We say that submanifolds $M_{1}, M_{2} \subset \mathbf{R}^{N}$ are a totally skew pair, if, for every $x \in M_{1}, y \in$ $M_{2}$, the tangent spaces $T_{x} M_{1}$ and $T_{y} M_{2}$ are skew. It follows immediately from Lemma 2.1 that $N \geq n_{1}+n_{2}+1$. The following result gives an improvement of this bound for closed submanifolds.

Theorem 6.2 If $M_{1}^{n_{1}}, M_{2}^{n_{2}} \subset \mathbf{R}^{N}$ are a totally skew pair of closed submanifolds, then $N \geq n_{1}+n_{2}+2$.

This lower bound is sharp, because there exists a totally skew pair in $\mathbf{R}^{n_{1}+n_{2}+2}=\mathbf{R}^{n_{1}+1} \times$ $\mathbf{R}^{n_{2}+1}$, given by

$$
M_{1}:=\mathbf{S}^{n_{1}} \times\{0\}, \quad \text { and } \quad M_{2}:=\{0\} \times \mathbf{S}^{n_{2}} .
$$

We offer two proofs for Theorem 6.2.

Proof 1. Since $M_{1} \cap M_{2}=\emptyset$, the (Gauss) map

$$
M_{1} \times M_{2} \ni(x, y) \stackrel{f}{\longmapsto} \frac{(x-y)}{\|x-y\|} \in \mathbf{S}^{N-1}
$$


is well-defined. Recall that the differential of $f$, evaluated at a tangent vector $(v, w)$ in $\bar{T}_{\left(x_{0}, y_{0}\right)}\left(M_{1} \times M_{2}\right)$, is given by

$$
d f_{\left(x_{0}, y_{0}\right)}((v, w)):=\left.\frac{d}{d t} f(x(t), y(t))\right|_{t=0},
$$

where $t \mapsto(x(t), y(t))$ is a curve in $M_{1} \times M_{2}$ with $(x(0), y(0))=\left(x_{0}, y_{0}\right)$ and $\left(x^{\prime}(0), y^{\prime}(0)\right)=$ $(v, w)$. Further note that the definition of $f$ yields

$$
\frac{d}{d t} f(x, y)=\frac{\left(x^{\prime}-y^{\prime}\right)\|x-y\|-(x-y)\left\langle x^{\prime}-y^{\prime}, x-y\right\rangle /\|x-y\|}{\|x-y\|^{2}} .
$$

Now suppose that $d f_{\left(x_{0}, y_{0}\right)}((v, w))=0$. Then the numerator of the above fraction vanishes at $t=0$, and we obtain

$$
v-w=k\left(x_{0}-y_{0}\right),
$$

for some scalar $k$. If $k \neq 0$, then $x_{0}-v / k=y_{0}-w / k$, and hence $T_{x_{0}} M_{1}$ intersects $T_{y_{0}} M$ which is a contradiction. So $k=0$, which yields $v=w$. Since $M_{1}$ and $M_{2}$ are a totally skew pair, this can happen only when $(v, w)=(0,0)$. So we conclude that $d f$ is non-singular everywhere. In particular, $N-1 \geq n_{1}+n_{2}$. Thus to complete the proof it remains to show that $N-1 \neq n_{1}+n_{2}$.

Suppose that $N-1=n_{1}+n_{2}$. Then, since $d f$ is non-singular, $f$ is a local homeomorphism. Thus, since $M_{1} \times M_{2}$ is compact, it follows that $f$ is onto, and is therefore a covering map. But $\mathbf{S}^{N-1}$ is simply connected (assuming $n_{1}, n_{2} \neq 0$ ), so it admits no non-trivial coverings. This means that $f$ has to be one-to-one, and hence a homeomorphism. Thus we obtain a contradiction, because, by basic homology theory, a product of manifolds cannot be homeomorphic to a sphere.

The next argument is more elementary:

Proof 2. Let conv $M_{1}$ and conv $M_{2}$ denote the convex hulls of $M_{1}$ and $M_{2}$, respectively. First suppose that conv $M_{1}=\operatorname{conv} M_{2}$. Let $p_{1}$ be a point of $M_{1}$ in the boundary of conv $M_{1}$, and $H \subset \mathbf{R}^{N}$ be a supporting hyperplane of $M_{1}$ through $p_{1}$. Then $H$ also supports $M_{2}$, and $H \cap \operatorname{conv}\left(M_{2}\right) \neq \emptyset$; therefore, $H$ must intersect $M_{2}$ at some point $p_{2}$. Thus $T_{p_{1}} M_{1}$ and $T_{p_{2}} M_{2}$ both lie in $H \simeq \mathbf{R}^{N-1}$, and Lemma 2.1 yields that $N-1 \geq n_{1}+n_{2}+1$, as desired.

So it remains to consider the case when conv $M_{1} \neq \operatorname{conv} M_{2}$. Then one of our manifolds, say $M_{1}$, must have a point, say $p_{0}$, which lies outside the convex hull of another. In particular, $M_{2}$ must have a support hyperplane $H$ which separates $M_{2}$ from $p_{0}$. We may move $H$ parallel to itself and away from conv $M_{2}$ until it becomes tangent to $M_{1}$, say at a point $p_{1}$. Since $\operatorname{dim}\left(T_{p_{1}} M_{1}\right)<\operatorname{dim}(H)$, we may "rotate" $H$ around $T_{p_{1}} M_{1}$ until it touches conv $M_{2}$ at some point $p_{2}$. So once again we obtain a support hyperplane for $M_{1} \cup M_{2}$ which intersects each submanifold, and we may invoke Lemma 2.1 to complete the proof.

\section{References}

1. Astey, L.: Geometric dimension of bundles over real projective spaces. Quart. J. Math. 31, 139-155 (1980)

2. Boltyansky, V., Ryzhkov, S., Shashkin, Yu.: On $k$-regular embeddings and on applications to theory of function approximation. Russ. Math. Surv. 15(6), 125-132 (1960)

3. Borsuk, K.: On the $k$-indipendent subsets of the Euclidean space and of the Hilbert space. Bull. Acad. Pol. Sci. Cl. III 5(4) (1957)

4. Chisholm, M.: $k$-regular mappings of $2^{n}$-dimensional Euclidean space. Proc. Am. Math. Soc. 74, 187190 (1979) 
5. Cohen, F., Handel, D.: $k$-regular embeddings of the plane. Proc. Am. Math. Soc. 72, 201-204 (1978)

6. Davis, D.: Generalized homology and the generalized vector field problem. Quart. J. Math. 25, 169-193 (1974)

7. Davis, D.: Desuspensions of stunted projective spaces. Pacif. J. Math. 113, 35-49 (1984)

8. Davis, D.: A strong non-immersion theorem for real projective spaces. Ann. Math. 120, 517-528 (1984)

9. Davis, D.: Immersions of projective spaces: a historical survey. Contemp. Math. 146, 31-37 (1993)

10. Davis, D., Gitler, S., Mahowald, M.: The stable geometric dimension of vector bundles over real projective spaces. Trans. Am. Math. Soc. 268, 39-61 (1981)

11. Eliashberg, Y., Mishachev, N.: Introduction to the $h$-principle. AMS, Providence (2002)

12. Farber, M., Tabachnikov, S., Yuzvinsky, S.: Topological robotics: motion planning in projective spaces. Int. Res. Math. Notes 34, 1853-1870 (2003)

13. Ghomi, M.: Shadows and convexity of surfaces. Ann. Math. 155(2), 281-293 (2002)

14. Ghomi, M.: Tangent bundle embeddings of manifolds in Euclidean space. Comm. Math. Helv. 81, 259-270 (2006)

15. Ghomi, M., Solomon, B.: Skew loops and quadratic surfaces. Comm. Math. Helv. 77, 767-782 (2002)

16. Ghomi, M.: Nonexistence of skew loops on ellipsoids. Proc. Amer. Math. Soc. 133, 3687-3690 (2005)

17. Golubitsky, M., Guillemin, V.: Stable mappings and their singularities. Springer, Heidelberg (1973)

18. Handel, D.: Algebraic-topological problems in approximation theory. Geometric applications of homotopy theory, Lect. Notes Math. 657, 267-270, (1978)

19. Handel, D.: Obstructions to 3-regular embeddings. Houston J Math. 5, 339-343 (1979)

20. Handel, D.: Some existence and nonexistence theorems for $k$-regular maps. Fund. Math. 109, 229-233 (1980)

21. Handel, D.: 2k-regular maps on smooth manifolds. Proc. Am. Math. Soc. 124, 1609-1613 (1996)

22. Handel, D., Segal, J.: On $k$-regular embeddings of spaces in Euclidean space. Fund. Math. 106, 231-237 (1980)

23. Hirsch, M.W.: Differential topology. Springer, Heidelberg (1994)

24. James, I.: Euclidean models of projective spaces. Bull. Lond Math. Soc. 3, 257-276 (1971)

25. Lai, H.F.: On parallel tangents of embeddings in codimension 2 in Euclidean spaces. Indiana Univ. Math. J. 22, 1171-1181 (1973)

26. Lam, K.Y.: Sectioning vector bundles over real projective spaces. Quart. J. Math. 23, 97-106 (1972)

27. Lam, K.Y., Randall, D.: Geometric dimension of bundles on real projective spaces. Contemp. Math., vol. 188, AMS, New York, 137-160 (1995)

28. Schneider, R.: Convex bodies: the Brunn-Minkowski theory encyclopedia of mathematics and its applications, vol. 44. Cambridge University Press, Cambridge (1993)

29. Segre, B.: Global differential properties of closed twisted curves. Rend. Sem. Mat. Fis. Milano 38, 256-263 (1968)

30. Sha, J.-P., Solomon, B.: No skew branes on non-degenerate hyperquadrics. Preprint, ArXiv: math.DG/ 0212197

31. Stojanovic, G.: Embeddings With Multiple Regularity. Geom. Dedicata. 123, 1-10 (2006)

32. Stojanovic, G., Tabachnikov, S.: Non-existence of $n$-dimensional $T$-embedded discs in $\mathbf{R}^{2 n}$. Comm. Math. Helv. 81, 877-882 (2006)

33. Tabachnikov, S.: On skew loops, skew branes and quadratic hypersurfaces. Moscow Math. J. 3, 681-690 (2003)

34. Tabachnikov, S., Tyurina, Y. Existence and non-existence of skew branes. Preprint, ArXiv: math.DG/ 0504484

35. Vassiliev, V.A.: Topology of complements to discriminants. (in Russian), Fazis, Moscow (1997)

36. White, J.: Global properties of immersions into Euclidean spheres. Indiana Univ. Math. J. 20, 1187-1194 (1971)

37. Whitney, H.: The self-intersections of a smooth $n$-manifold in $2 n$-space. Ann. of Math. 45, 220-246 (1944)

38. Wu, Y.-Q.: Knots and links without parallel tangents. Bull. Lond Math. Soc. 34, 681-690 (2002) 